\title{
The Molecular Defect in Type IIB von Willebrand Disease Identification of Four Potential Missense Mutations within the Putative Gplb Binding Domain
}

\author{
Kathleen A. Cooney," William C. Nichols," Mary E. Bruck, ${ }^{*}$ Wadie F. Bahou," Amy D. Shapiro," \\ E. J. Walter Bowie," Harvey R. Gralnick, ${ }^{\star *}$ and David Ginsburg** \\ ${ }^{\ddagger}$ Howard Hughes Medical Institute and Department of Human Genetics, University of Michigan Medical School, Ann Arbor, Michigan \\ 48109-0650; *Department of Internal Medicine, University of Michigan Medical School, Ann Arbor, Michigan 48109-0650, \\ 'Mayo Clinic and Mayo Foundation, Rochester, Minnesota 55905; "Indiana University Medical Center, Indianapolis, \\ Indiana 46202; ${ }^{* *}$ Hematology Service, National Institutes of Health, Bethesda, Maryland 20814; \\ and ${ }^{\S}$ Department of Medicine, State University of New York, Stony Brook, New York 11794
}

\begin{abstract}
Type IIB von Willebrand Disease (vWD) is characterized by the selective loss of large von Willebrand Factor (vWF) multimers from plasma, presumably due to their increased reactivity with platelets and subsequent clearance from the circulation. Using the PCR, one of a panel of four potential missense mutations was identified in each of the 14 patients studied from 11 unrelated families. None of these substitutions was encountered in a large panel of normal DNAs. These changes all represent $\mathrm{C} \rightarrow \mathrm{T}$ transitions at $\mathrm{CpG}$ dinucleotides, proposed "hot spots" for mutation in the human genome. The four resulting amino acid substitutions, Arg543 $\rightarrow$ Trp, Arg545 $\rightarrow$ Cys, Val553 $\rightarrow$ Met, and Arg578 $\rightarrow$ Gln, are all clustered within the GpIb binding domain of vWF. Disruption of this latter functional domain may explain the pathogenesis of Type IIB vWD. By sequence polymorphism analysis, the Arg543 $\rightarrow$ Trp substitution was shown to have occurred as at least two independent mutational events. This latter observation, along with the identification of mutations in all 14 patients studied and their localization to the GpIb binding domain, all strongly suggest that these substitutions represent the authentic defects responsible for Type IIB vWD. This panel of mutations may provide a useful diagnostic tool for the majority of patients with Type IIB vWD. (J. Clin. Invest. 1991. 87:1227-1233.) Key words: von Willebrand Factor • thrombocytopenia • ristocetin-induced platelet aggregątion • polymerase chain reaction • dideoxy sequencing
\end{abstract}

\section{Introduction}

von Willebrand Disease (vWD) ${ }^{1}$ is the most common inherited bleeding disorder in man with a prevalence estimated to be as high as $1 \%$ of the population (1). The disorder results from either a quantitative (Type I) or a qualitative (Type II) defect in

Address correspondence and reprint requests to Dr. David Ginsburg, Howard Hughes Medical Institute, University of Michigan Medical Center, 4520 MSRBI, 1150 West Medical Center Drive, Ann Arbor, MI 48109-0650.

Received for publication 1 October 1990.

1. Abbreviations used in this paper: ASO, Allele-specific oligonucleotide; GpIb, glycoprotein Ib; RFLP, restriction length polymorphism; RIPA, ristocetin-induced platelet aggregation; vWD, vWF, von Willebrand Disease, Factor.

J. Clin. Invest.

(c) The American Society for Clinical Investigation, Inc. $0021-9738 / 91 / 04 / 1227 / 07 \quad \$ 2.00$

Volume 87, April 1991, 1227-1233 von Willebrand Factor (vWF) and is typically transmitted in an autosomal dominant fashion. Type II vWD is identified by selective loss of plasma vWF high and/or intermediate molecular weight multimers (2). Type IIB vWD is additionally characterized by enhanced ristocetin-induced platelet aggregation (RIPA) and increased binding of the abnormal vWF to normal platelets $(3,4)$. Clinically, Type IIB vWD is associated with a bleeding disorder of variable severity and thrombocytopenia which is often exacerbated by pregnancy $(5,6)$, stress $(7)$, advanced age (8), or 1-deamino-8-D-arginine-vasopressin (DDAVP) (9). The latter, the treatment of choice for many forms of vWD, is relatively contraindicated in Type IIB (9).

Patients with Type IIB vWD specifically lack the largest multimers of vWF in plasma but not in platelets. It has been hypothesized that plasma high molecular weight vWF multimers of patients with Type IIB vWD bind spontaneously to platelets and are subsequently cleared from the circulation (10), accounting for both the characteristic vWF multimer pattern and the frequently associated thrombocytopenia. In support of this hypothesis, vWF secreted by Type IIB vWD umbilical vein endothelial cells were shown to contain the full range of vWF multimers on gel analysis and to induce spontaneous platelet aggregation in vitro (11).

The human vWF gene has been localized to the short arm of chromosome $12(12)$ and spans $178 \mathrm{~kb}$ interrupted by 51 introns (13). In addition, a nonprocessed pseudogene highly homologous to the mid-portion of VWF has been identified on human chromosome 22 (14). vWF is synthesized by endothelial cells and megakaryocytes and undergoes extensive posttranslational processing including multimerization prior to secretion (15). The 2813 residue vWF amino acid sequence consists of multiple repeated segments, including the triplicated A domains $(16,17)$. A region important for the interaction of vWF with platelet glycoprotein Ib (GpIb), one of the primary receptors for vWF on platelets, has been localized to the vWF Al domain between amino acids 449 and 728 of the mature protein (18). This region also contains binding sites for heparin (19) and collagen $(20,21)$. The enhanced platelet aggregation induced by vWF prepared from a patient with Type IIB vWD can be blocked by a monoclonal antibody directed against GpIb $(3,22)$. Furthermore, the tryptic fragment of normal vWF containing the GpIb binding domain (amino acids 449728) was shown to block spontaneous platelet aggregation in patients with the Tampa variant of Type IIB vWD (23).

Over the past several years, molecular defects responsible for several types of vWD have been identified. Large gene deletions appear to be an uncommon mechanism for vWD, seen primarily in patients with inhibitors to $\operatorname{VWF}(14,24,25)$. Missense mutations within the vWF A2 domain were recently 
identified in two patients with Type IIA vWD (26) and additional Type IIA patients may demonstrate other mutations in the same region (27). We now report the identification of four distinct potential vWF missense mutations occurring in 14 patients from 11 unrelated Type IIB vWD families. These substitutions are all contained in a 36-amino acid segment within the putative GpIb binding domain of vWF.

\section{Methods}

Patient material. 14 patients with Type IIB vWD from 11 unrelated families were studied (B1-14). The family of patient $\mathrm{B} 14$ has been previously reported (7). The father of B6 has also been reported (3). Patients were classified by referring physicians as having Type IIB vWD if they had a moderately severe bleeding disorder characterized by some or all of the following clinical features: thrombocytopenia, prolonged bleeding time, normal to low factor VIII procoagulant activity, normal to low vWF antigen, low plasma ristocetin cofactor activity, decreased circulating high molecular weight $\mathrm{vWF}$ multimers with intact platelet $\mathrm{vWF}$ multimers, enhanced RIPA at a low concentration of ristocetin $(0.2-0.6 \mathrm{mg} / \mathrm{ml})$, and the absence of platelet aggregation or agglutination when normal purified $\mathrm{vWF}$ or cryoprecipitate is added to patient platelet rich plasma. Genomic DNA was prepared from peripheral blood leukocytes by standard methods (12). Genomic DNA was also obtained from 67 normal individuals with no prior history of bleeding disorders. The umbilical cord from a patient with Type IIB vWD was also obtained and a human umbilical vein endothelial cell culture was prepared (28). DNA was isolated from the cultured cells and is included in subsequent analysis as B5. Clinical laboratory data obtained from the child are consistent with the diagnosis of Type IIB vWD.

DNA sequence analysis. The PCR was used to amplify vWF exon 28 sequences as previously described (26). Nucleotide position numbering is per the sequence of Bonthron, et al. (29), with the "A" of the initiator "ATG" codon taken as +1 . PCR primers designed to amplify $\sim 1.3 \mathrm{~kb}$ of genomic DNA containing exon 28 were primer A: CACGAATTCTACTGCAGCAGGCTACTGGACCTGGTCT, and either primer B: CACGGATCCGGGAGCGTCTCAAAGTCCTGGATGAGGATA, or primer C: CACGGATCCAGGGAAGCCAGGATTAGAACCCGAGTCG. Primer A corresponds to (+) strand nucleotides $3811-3838$ of the vWF cDNA sequence (29), primer B to vWF cDNA (-) strand sequence 4973-4944, and primer C to (-) strand sequence of intron 28 (nucleotide positions 24/1666-24/1695 according to Mancuso, et al.) ([13] and D. Ginsburg and M. Bruck, unpublished data). Primers were engineered to create synthetic restriction sites (underlined) at their $5^{\prime}$ ends. The technique of allele-specific PCR $(30,31)$ was used to selectively amplify the authentic vWF gene. Primers $B$ and $C$ were positioned such that their $3^{\prime}$ terminal nucleotides match the sequence of the authentic vWF gene but differ from that of the vWF chromosome 22 pseudogene ([26] and D. Ginsburg and M. Bruck, unpublished data). PCR conditions were as previously described (26). For B1, B3, and B12, PCR products were amplified with primers $A$ and $C$, digested with Eco RI and Bam $\mathrm{HI}$, and directionally subcloned into M13mp19. The entire 1215 bp of exon within the PCR product were sequenced with Sequenase (United States Biochemical, Cleveland, $\mathrm{OH}$ ) from multiple independent clones either as pooled sequencing reaction (B3 and $\mathrm{B} 12$ ) or as individual clones (B1 and $\mathrm{B} 12$ ), as previously described (26). Mutations first identified in pooled sequence (B3) were confirmed by analysis of individual clones. DNA from patient $B 6$ was analyzed from nucleotide 3820 to 4120 by direct sequence of PCR products using asymmetric PCR (32) as previously described (33). The mutations identified by allele-specific oligonucleotide (ASO) or restriction digest for patients B2, B4, B5, B7, B8, B9, B10, $B 11, B 12$, and $B 14$ were all confirmed by asymmetric PCR sequencing. Asymmetric PCR was performed with replinase (New England Nuclear, Bannockburn, IL).
Restriction analysis and allele-specific oligonucleotide hybridization. Cellular DNA was amplified by PCR using primers A and B, as described above. The C3922 $\rightarrow \mathrm{T}$ and G4022 $\rightarrow$ A substitutions both result in convenient restriction site changes, loss of an Hha I site and gain of a Pst I site, respectively. For analysis of these substitutions, PCR products were digested with Hha I or Pst I (Bethesda Research Laboratories, Gaithersburg, MD) and analyzed by fractionation on an ethidium bromide-stained $4 \%$ composite agarose gel (3\% NuSieve agarose [FMC BioProducts, Rockland ME] and $1 \%$ standard agarose [Bethesda Research Laboratories]). The remaining substitutions were evaluated by dot blot analysis and ASO hybridization, as previously described (34). 15-mer oligonucleotides were synthesized with either normal (underlined) or "mutant" (shown in parentheses) sequence in the 8th position. Probes 1 and 2: GATGGAGC(T)GGCTGCG; probes 3 and 4: GGTCCGCG(A)TGGCCGT; probes 5 and 6: TTTGTCCG(A)CTACGTC. Probes 2, 4, and 6 are the "mutant" oligonucleotides corresponding, respectively, to the normal sequences of probes 1,3 , and 5 (see Fig. 1). $3 \mu \mathrm{l}$ of PCR product were denatured in $200 \mu \mathrm{l}$ of 0.4 $\mathrm{N}$ sodium hydroxide, $25 \mathrm{mM}$ EDTA, and applied to a nylon filter (Hybond-N; Amersham Corp., Arlington Heights, IL) using a dot blot apparatus (Hybri - dot; Bethesda Research Laboratories). Filters were hybridized with end-labeled oligonucleotides with the final wash performed in $5 \times$ SSPE and $0.1 \%$ SDS for $10 \mathrm{~min}$ at an optimal temperature empirically determined for each ASO probe.

\section{Results}

Exon 28 sequence in patient B1. B1 DNA was amplified with primers $A$ and $C$ and subcloned into M13. Initial sequence analysis demonstrated that the patient is heterozygous for a common polymorphism at nucleotide 4641 in the vWF coding sequence. This polymorphism results in the presence $(+)$ or absence (-) of a Bst EII restriction site (Nichols, W. C., S. E.

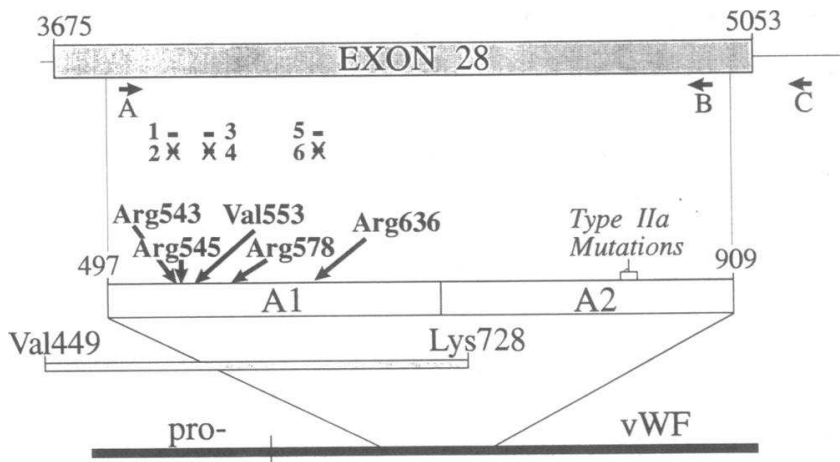

Figure 1. Location of PCR and ASO primers. Exon 28, containing coding sequence nucleotides $3675-5053$ (29), is shown schematically at the top with the location and orientation of PCR primers A, B, and $C$ indicated by arrows. The corresponding amino acid sequence from mature subunit residues 497-909 are shown below, including two of the three triplicated A domains $(A 1$ and $A 2)(16,17)$ and the location of Arg543, Arg545, Val553, Arg578, and Arg636. The segment containing the previously reported Type IIA vWD mutations is also indicated (26). The locations of the ASO oligonucleotide pairs $1 / 2,3 / 4$, and $5 / 6$, designed to detect the nucleotide substitutions at Arg543, Val553, and Arg636, respectively, are also illustrated. Oligonucleotides corresponding to the mutant sequences are depicted with an $X$. The position of this region within the full length vWF coding sequence is shown at the bottom along with the location of the tryptic fragment Val449-Lys728, containing the putative Gplb binding domain (18). 
A.

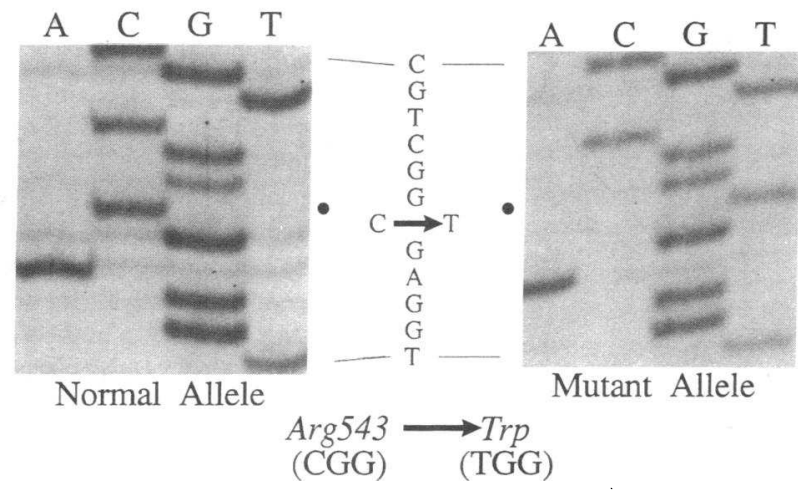

B.

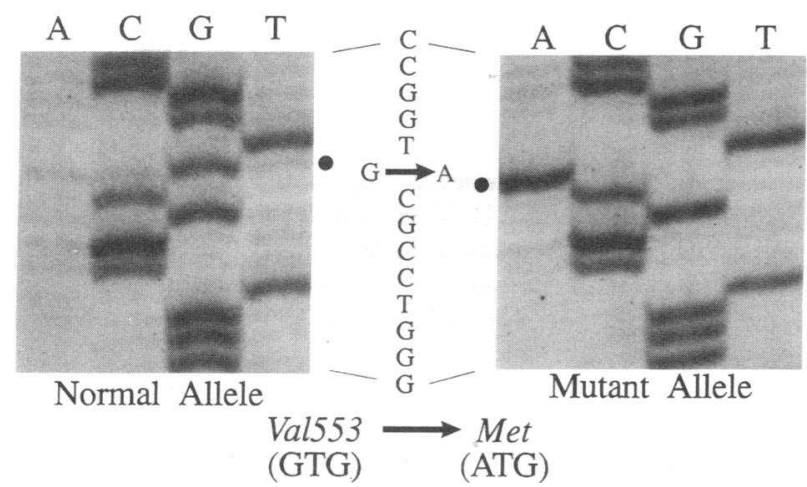

Figure 2. Sequence of Type IIB vWD mutations. Sequence analysis of individual M1 3 clones containing normal (left) and mutant (right) sequences are shown. The positions of nucleotide substitutions are indicated by filled circles. $(A)$ The $\mathrm{C} \rightarrow \mathrm{T}$ substitution at nucleotide 3916 in patient B1 results in the substitution of Trp for Arg543. (B) The $\mathrm{G} \rightarrow \mathrm{A}$ transition at nucleotide 3946 in patient $\mathrm{B} 12$ results in the substitution of Met for Val553.

Lyons, D. Ginsburg, manuscript in preparation). Individual M13 exon 28 PCR clones were typed for this restriction fragment length polymorphism (RFLP) and the inserts from two independent clones for each allele were sequenced in their entirety. In this way authentic sequence differences, which should be present in both clones of the same allele, could be distinguished from PCR errors, which should be present in only one clone. Only normal sequence was observed on the Bst EII (-) allele, whereas two novel changes, both resulting in amino acid substitutions, were identified on the Bst EII $(+)$ allele: a C $\rightarrow$ T transition at cDNA nucleotide 3916 resulting in an Arg543 $\rightarrow$ Trp substitution (Fig. $2 A$ ) and a G $\rightarrow$ A transition at nucleotide 4196 resulting in an Arg636 $\rightarrow$ His substitution (data not shown).

Identification of a rare sequence polymorphism at nucleotide 4196. DNA from 67 normal individuals and the 14 Type IIB vWD patients were amplified with primers $A$ and $B$, and analyzed by dot blot analysis and ASO hybridization using ASO probes 5 and 6. Patient B1, two of the normal individuals, and none of the 13 additional Type IIB vWD patients were found to be heterozygous for the G4196 $\rightarrow$ A transition (Fig. 3,
$C$ and $D$ ). Thus this nucleotide difference, resulting in an Arg636 $\rightarrow$ His amino acid substitution, represents a rare coding sequence polymorphism with an allele frequency of $\sim 0.015$.

Identification of a potential Type IIB $v W D$ mutation at nucleotide 3916. The remaining 13 Type IIB vWD patients as well as 67 normal patients were screened for the presence of the nucleotide 3916 substitution, using ASO probes 1 and 2. This substitution was not detected in any of the 134 normal vWF alleles screened. However, when the additional 13 Type IIB vWD patients were screened, 3 were observed to have the identical C $\rightarrow$ T substitution at nucleotide 3916 (B4, B5, and B9; see Fig. $3 A, B)$. B4 is the mother of B5. Direct DNA sequence analysis by asymmetric PCR confirmed the ASO genotype data.

Identification of a second potential Type IIB $v W D$ mutation at nucleotide 3946 . For B12 DNA, sequence analysis was performed for both pooled and individual M13 clones as described above. A single change, a $\mathrm{G} \rightarrow \mathrm{A}$ transition at nucleotide 3946 , was identified in one allele, resulting in a Val553 $\rightarrow$ Met substitution (Fig. $2 \mathrm{~B}$ ). The same potential missense mutation was detected by dot blot analysis and ASO hybridization, using ASO probes 3 and 4 in the patient's affected son (B13) and in two other unrelated Type IIB vWD patients (B2 and B8; Fig. 4), but was not detected in any of 98 normal alleles screened (data not shown). The ASO data were confirmed by direct DNA sequence analysis of the PCR products, as described above.

Identification of a third potential Type IIB $v W D$ mutation at nucleotide 3922. B3 DNA was amplified with primers A and $\mathrm{C}$ and the resulting PCR products sequenced as a pool of M13 clones. A single substitution, a $\mathrm{C} \rightarrow \mathrm{T}$ transition at nucleotide 3922 was identified and confirmed by sequence analysis of individual M13 clones (Fig. $5 \mathrm{~A}$ ). This change, which results in an Arg545 $\rightarrow$ Cys substitution, destroys an Hha I restriction site and thus can be detected by PCR and restriction enzyme digestion (Fig. 5 B). By this analysis, patients B7, B10, B1 1, and B14 were all found to have the same substitution. B11 is the son of B10. This change was not identified in any of 98 normal vWF alleles analyzed.

Identification of a fourth potential Type IIB $v W D$ mutation at nucleotide 4022 . By the above analysis, potential mutations were identified in 13 of the 14 Type IIB vWD patients studied. Sequence analysis in the vicinity of these mutations in the remaining patient, B6, identified a novel substitution, a $G \rightarrow A$ transition at nucleotide 4022 . This change results in an amino acid substitution of Gln for Arg578. This mutation results in the creation of a new Pst I site at this position, and thus could be confirmed by PCR and restriction enzyme analysis (Fig. 6). This substitution was not observed in any of the other Type IIB vWD patients or in any of 98 normal vWF alleles screened.

\section{Discussion}

Surveying the vWF gene for potential point mutations associated with the many distinct $v W D$ phenotypes poses a formidable task, given the enormous size and complexity of the gene and limited availability of vWF mRNA. To date, such mutations have only been successfully identified for the Type IIA vWD variant. For this latter phenotype, the responsible missense mutations appear to be clustered in a small region within the $A 2$ repeat of $\mathrm{vWF}$. There appears to be an increased sensitiv- 


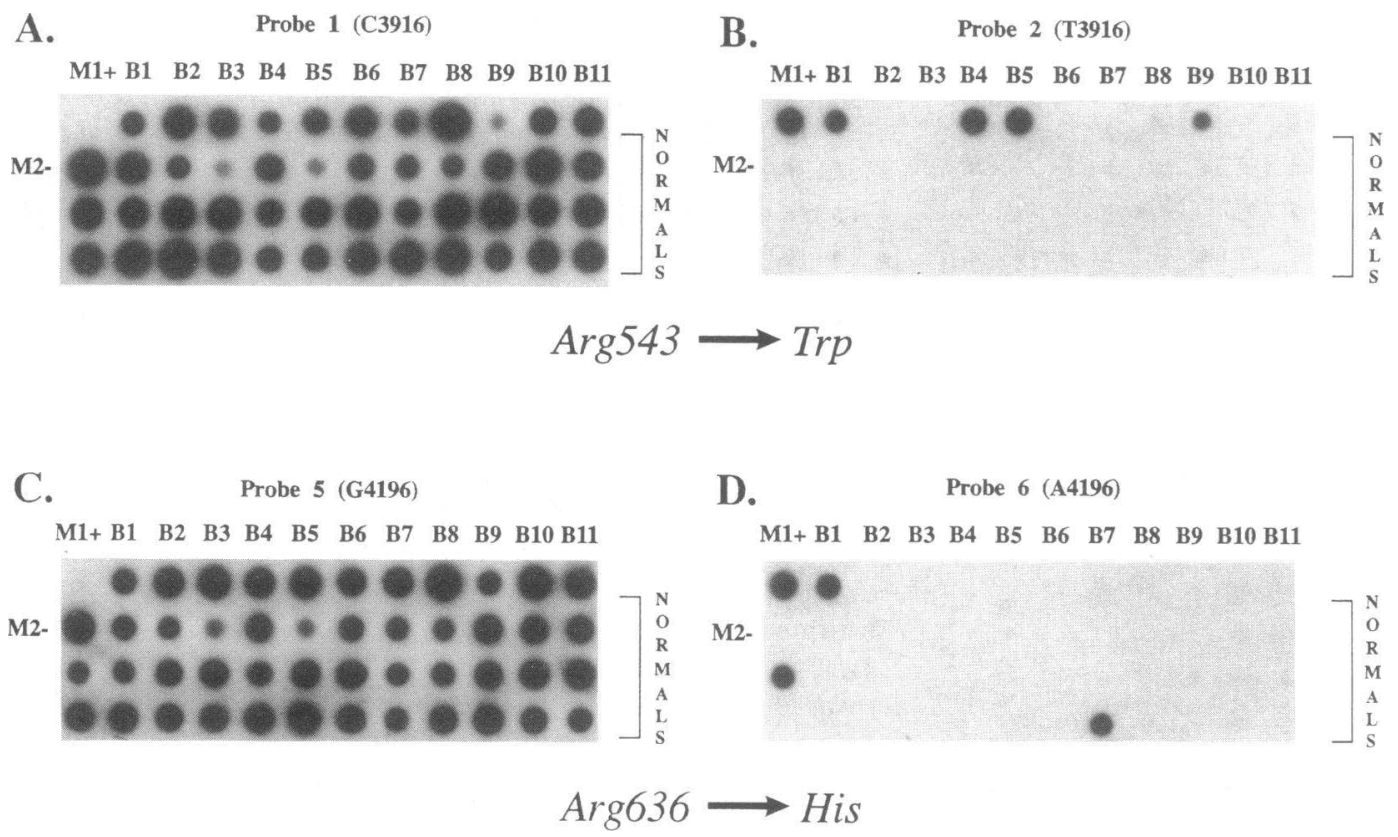

Figure 3. PCR ASO analysis of the C3916 $\rightarrow$ T mutation and the G4196 $\rightarrow$ A polymorphism. DNA was amplified by PCR and applied to four identical nylon filters. Each filter was probed with an ASO primer as indicated (Probe $1,2,5$, or 6 ). $A$ and $B$ show genotype analysis for the Arg543 $\rightarrow$ Trp mutation at 3916. $C$ and $D$ show genotype analysis for the Arg636 $\rightarrow$ His polymorphism at 4196 . The location of samples corresponding to patients B1B11 are indicated. PCR products from individual M13 clones were used as hybridization controls. M13 clone M1 contains both the mutant sequence at 3916 and the rare polymorphism at 4196 (designated by a '+'). M13 clone M2 contains the normal sequence at 3916 and the more common sequence at 4196 (designated by a '-'). 36 normal DNA samples are also shown. Individuals B1, B4, B5, and B9 are all seen to be heterozygous for the mutation at 3916. Individuals B2, B3, B6, B7, B8, B10, and B11 as well as all 35 normal individuals are seen as homozygous normal $(A$ and $B)$. Individual B1 and two normals are seen as heterozygous for the rare polymorphism at 4196 while all other patients and normals are homozygous for the more common allele $(C$ and $D$ ). Individuals B12, B13, and B14 are all homozygous for both the normal allele at 3916 and the more common allele at 4196 (not shown).

ity to proteolysis in this region of Type IIA vWF associated with a characteristic increase in the corresponding vWF proteolytic fragment in plasma. This latter observation provided an important localizing clue for the identification of Type IIA vWD mutations $(26,27)$.

The Type IIB vWD phenotype is associated with a loss of large vWF multimers similar to that of Type IIA, but occurring through a unique mechanism related to an apparent increased affinity for the platelet $\mathrm{vWF}$ receptor $\operatorname{GpIb}(3,11,22,23)$. The vWF functional domain responsible for GpIb binding appears to reside between Val449 and Lys728 $(18,35)$. Based on the hypothesis that missense mutations within this region might alter the vWF/GpIb interaction resulting in the Type IIB vWD phenotype, a portion of vWF exon 28, encoding Lys534Pro921, was targeted for DNA sequence analysis. Four potential missense mutations, resulting in Arg543 $\rightarrow$ Trp, Arg545 $\rightarrow$ Cys, Val553 $\rightarrow$ Met, and Arg578 $\rightarrow$ Gln substitutions, were identified by this approach, each occurring in a different Type IIB vWD patient. The first three substitutions were subsequently detected by ASO hybridization analysis or restriction enzyme digestion in additional unrelated Type IIB vWD patients. For each of these three mutations, inheritance of the same sequence could also be traced to an affected offspring (B4 and B5 for Arg543 $\rightarrow$ Trp, B10 and B11 for Arg545 $\rightarrow$ Cys, and $B 12$ and B13 for Val553 $\rightarrow$ Met). In total, the four identified mutations account for all 14 Type IIB vWD patients studied, derived from 11 unrelated families.

A rare DNA sequence polymorphism at nucleotide 4196 was identified in patient $B 1$, on the same allele as the candidate Arg543 $\rightarrow$ Trp mutation. The two other Arg543 $\rightarrow$ Trp patients were homozygous for the more common allele at position 4916. The association of this same substitution with at least two distinct chromosomal backgrounds (4916 [+] and 4916 [-]) strongly suggests that this particular mutation has arisen at least two independent times. The occurrence of the Arg543 $\rightarrow$ Trp substitution as at least two independent mutational events in three Type IIB patients, with its absence from the 134 normal chromosomes screened, provides compelling genetic evidence that this substitution represents an authentic Type IIB vWD mutation. The identification of three other amino acid substitutions in eight other Type IIB vWD families, all also within the vWF GpIb binding domain (see below), provides further evidence in support of this hypothesis, as does the appropriate inheritance of the mutant alleles in three affected offspring. Whether the Arg545 $\rightarrow$ Cys and Val553 $\rightarrow$ Met mutations arose via more than one independent mutational event and whether two or three independent events occurred among the Arg543 $\rightarrow$ Trp families must await more detailed vWF RFLP haplotype analysis. Of note, all four potential missense mutations reported here represent $\mathrm{C} \rightarrow \mathrm{T}$ transitions at $\mathrm{CpG}$ dinucleotides, proposed "hot spots" for mutation in the human genome (36). None of these mutations are contained in vWF pseudogene sequence (Ginsburg, D., and M. Bruck, unpublished data).

All four mutations are contained in a 36 amino acid segment within the vWF tryptic fragment Val449 $\rightarrow$ Lys728, which has been shown to interact with platelet GpIb, heparin, and collagen (18-21, 37). Mohri, et al. have further localized GpIb binding to two discontinuous segments within this region (Lys474-Pro488 and Leu694-Pro708), and speculate that, in the intact molecule, disulfide bonds at 471-474 and 509-695 (38) may maintain these segments in close proximity (35). The four Type IIB substitutions, located within the region connecting these two segments, may all alter this critical vWF/GpIb 
A.

Probe 3 (G3946)

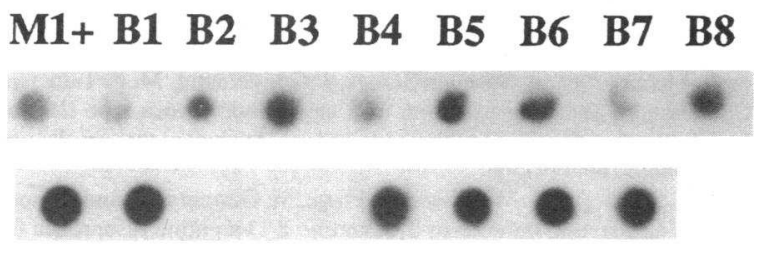

M2+ M3+ M4-M5- B12 B13 B2 B3

B.

Probe 4 (A3946)

M1+ B1 $\begin{array}{lllllll}\text { B2 } & \text { B3 } & \text { B4 } & \text { B5 } & \text { B6 } & \text { B7 } & \text { B8 }\end{array}$

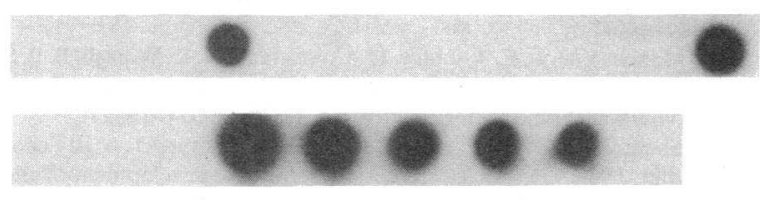

M2+ M3+ M4-M5- B12 B13 B2 B3

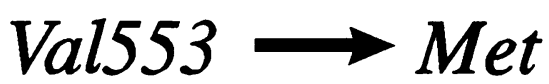

Figure 4. PCR ASO analysis of the G3946 $\rightarrow$ A mutation. DNA was amplified by PCR and bound to duplicate nylon filters. Each filter was probed with an ASO primer specific for either the normal allele (probe $3 ; A$ ) or the mutant allele (probe $4 ; B$ ). Patient samples (B1-B8, $\mathrm{B} 12, \mathrm{~B} 13)$ are identified as in the text. PCR products from individual M13 clones were used as hybridization controls. M13 clones M1, M2, and $M 3$ contain the normal sequence (designated by a ' + ') and M13 clones M4 and M5 contain the mutant sequence (designated by a '-'). Individuals B2 (in duplicate), B8, B12, and B13 are all seen to be heterozygous for the mutant allele. Individuals B1, B3 (in duplicate), B4, B5, B6, and B7 are seen to be homozygous normal. Individuals B9, B10, B11, and B14 are also homozygous normal (not shown).

interaction, resulting in the Type IIB vWD phenotype. The vWF segment from Leu $512 \rightarrow$ Lys673, which again contains all four Type IIB substitutions, may also play an important role in vWF's interaction with collagen and glycosoaminoglycans (37). Plasma vWF does not bind spontaneously to platelets, and initial binding to collagen and/or other subendothelial matrix components may be the critical in vivo trigger which subsequently permits binding to GpIb. The Type IIB vWD mutations could conceivably induce a conformational change in $\mathrm{vWF}$, mimicking the affect of matrix or collagen binding, and thereby leading to the pathologic spontaneous platelet binding characteristic of Type IIB vWD. Of note, these four Type IIB vWD mutations are localized within a highly basic region (11 basic residues within this 36 amino acid segment) and three of the four changes alter an Arg residue. Electrostatic interactions may be critical for $v W F / G p I b$ binding and may explain the facilitory effect of ristocetin on the binding of wild-type vWF to GpIb and the reactivity of Type IIB vWF in the absence of
A.

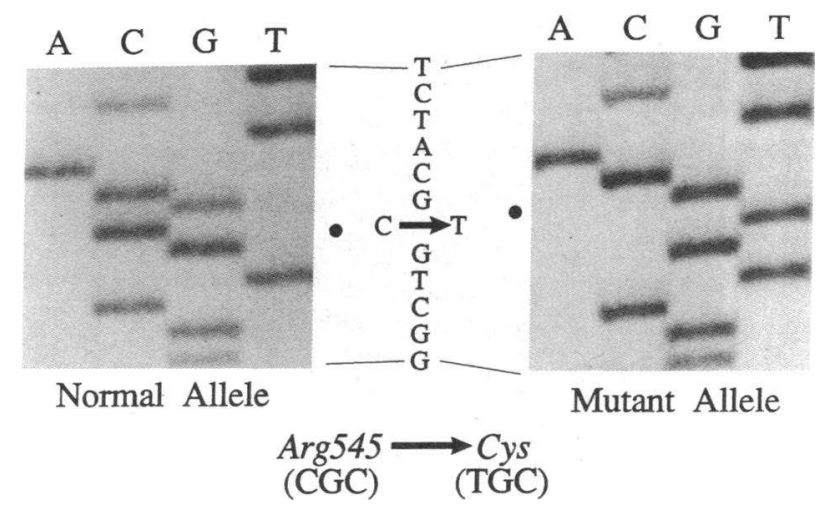

B.

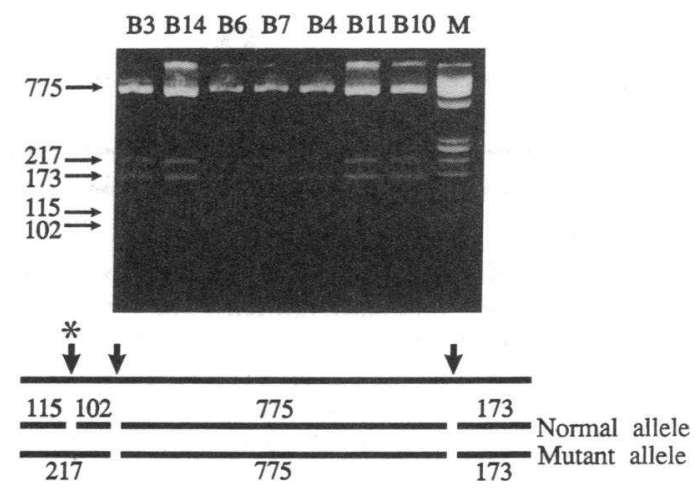

Figure 5. Analysis of the $\mathrm{C} 3922 \rightarrow \mathrm{T}$ mutation. $(A)$ Sequence analysis of individual M13 clones containing normal (left) and mutant (right) sequences from patient B3 are shown. The filled circles mark the $\mathrm{C} \rightarrow \mathrm{T}$ substitution at nucleotide 3922 , which results in the substitution of Cys for Arg545. (B) PCR-RFLP analysis for direct detection of the C3922 $\rightarrow \mathrm{T}$ mutation is shown. DNA was amplified, restricted with $\mathrm{Hha} I$ and fractionated on a $4 \%$ agarose gel. Patient samples are as identified in the text. The marker (lane $M$ ) is an Hae III digest of $\phi \mathrm{X} 174 \mathrm{DNA}$. The $\mathrm{C} \rightarrow \mathrm{T}$ substitution results in the loss of an Hha I site, as shown schematically at the bottom. The location of the three Hha I sites in exon 28 are marked by arrows with the site lost by the mutation indicated by an asterisk. The expected Hha I fragments for both the normal and mutant alleles are indicated. After Hha I digestion heterozygotes for the mutant allele demonstrate a novel 217-bp fragment. Individuals B3, B14, B7, B10, and B11 are seen to be heterozygous for the mutant allele. Individuals B6 and B4 are homozygous normal.

ristocetin. Confirmation of this hypothesis must await further functional studies.

This apparent clustering of Type IIB vWD mutations within the vWF Al domain is reminiscent of the similar localization of Type IIA mutations within the adjacent A2 repeat. These observations may provide important insights into vWF structure and function. In addition, the identification of potential defects in all 11 families studied suggests that this panel of mutations may offer a precise DNA diagnostic tool applicable to the vast majority of Type IIB vWD patients. This approach should be of particular value in Type IIB where classification has a major impact on clinical management (9). These findings together with similar work in the remaining subtypes, may eventually lead to a comprehensive DNA-based classification system for $\mathrm{vWD}$. 

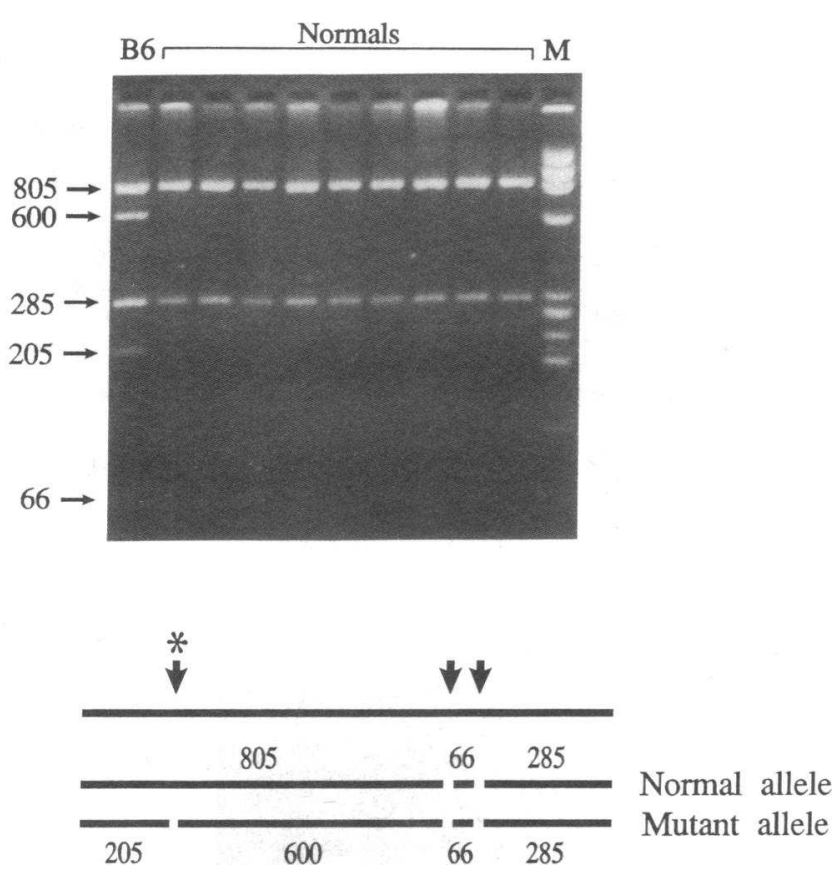

Figure 6. PCR-RFLP analysis for direct detection of the G4022 $\rightarrow$ A mutation. DNA was amplified, restricted with Pst I, and fractionated on a $4 \%$ agarose gel. The patient sample and normals are as identified in the text. The marker (lane $M$ ) is a Hae III digest of $\phi$ X174 DNA. The $G \rightarrow A$ substitution results in the creation of a Pst I site, as shown schematically at the bottom. The Pst I sites are marked by arrows and the site created by the mutation is indicated by an asterisk. After Pst I digestion heterozygotes for the mutant allele demonstrate novel fragments of 600 and $205 \mathrm{bp}$. Individual B6 is seen to be heterozygous for the mutant allele while the normal controls in the remaining lanes are homozygous normal. This mutation results in the substitution of Gln for Arg578.

\section{Acknowledgments}

We thank S. Labun for preparation of the manuscript, S. Norton for oligonucleotide synthesis, S. Weiss for assistance in establishing primary endothelial cell cultures, B. Konkle for identification of one of the Type IIB vWD families, P. Bockenstedt for helpful advice and thoughtful review of the manuscript, M. D. Benson for contributing the panel of normal DNA samples, and J. E. Sadler for sharing data before publication.

This work was supported in part by National Institutes of Health grant 5R01 HL-39693-02. Dr. Ginsburg is a Howard Hughes Medical Institute Associate Investigator.

\section{References}

1. Rodeghiero, F., G. Castaman, and E. Dini. 1987. Epidemiological investigation of the prevalence of von Willebrand's Disease. Blood. 69:454-459.

2. Ruggeri, Z. M., and T. S. Zimmerman. 1987. von Willebrand factor and von Willebrand Disease. Blood. 70:895-904.

3. Gralnick, H. R., S. B. Williams, L. P. McKeown, M. E. Rick, P. Maisonneuve, C. Jenneau, and Y. Sultan. 1985. Von Willebrand's disease with spontaneous platelet aggregation induced by an abnormal plasma von Willebrand factor. J. Clin. Invest. 76:1522-1529.

4. Ruggeri, Z. M., F. I. Pareti, P. M. Mannucci, N. Ciavarella, and T. S Zimmerman. 1980. Heightened interaction between platelets and factor VIII/ von Willebrand factor in a new subtype of von Willebrand's disease. $N$. Engl. J. Med. 302:1047-1051.

5. Rick, M. E., S. B. Williams, R. A. Sacher, and L. P. McKeown. 1987. Thrombocytopenia associated with pregnancy in a patient with Type IIB von Willebrand's disease. Blood. 69:786-789.
6. Giles, A. R., H. Hoogendoorn, and K. Benford. 1987. Type IIB von Willebrand's disease presenting as thrombocytopenia during pregnancy. $\mathrm{Br}$. J. Haematol. 67:349-353.

7. Hultin, M. B., and I. I. Sussman. 1990. Postoperative thrombocytopenia in Type IIB von Willebrand disease. Am. J. Hematol. 33:64-68.

8. Mazurier, C., A. Parquet-Gernez, J. Goudemand, M. F. Taillefer, and M. Goudemand. 1988. Investigation of a large kindred with type IIB von Willebrand's disease, dominant inheritance and age-dependent thrombocytopenia. $\mathrm{Br}$. J. Haematol. 69:499-505.

9. Holmberg, L., I. M. Nilsson, L. Borge, M. Gunnarsson, and E. Sjorin. 1983. Platelet aggregation induced by 1-desamino-8-D-arginine vasopressin (DDAVP) in Type IIB von Willebrand's disease. N. Engl. J. Med. 309:816-821.

10. Ruggeri, Z. M., R. Lombardi, L. Gatti, R. Bader, C. Valsecchi, and T. S. Zimmerman. 1982. Type IIB von Willebrand's disease: differential clearance of endogenous versus transfused large multimer von Willebrand factor. Blood. 60:1453-1456

11. de Groot, P. G., A. B. Federici, H. C. de Boer, P. d'Alessio, P. M. Mannucci, and J. J. Sixma. 1989. von Willebrand factor synthesized by endothelial cells from a patient with Type IIB von Willebrand disease supports platelet adhesion normally but has an increased affinity for platelets. Proc. Natl. Acad. Sci. USA. 86:3793-3797.

12. Ginsburg, D., R. I. Handin, D. T. Bonthron, T. A. Donlon, G. A. P. Bruns, S. A. Latt, and S. H. Orkin. 1985. Human von Willebrand Factor (VWF): isolation of cDNA clones and chromosomal localization. Science (Wash. DC) 228:1401-1406.

13. Mancuso, D. J., E. A. Tuley, L. A. Westfield, N. K. Worrall, B. B. SheltonInloes, J. M. Sorace, Y. G. Alevy, and J. E. Sadler. 1989. Structure of the gene for human von Willebrand factor. J. Biol. Chem. 264:19514-19527.

14. Shelton-Inloes, B. B., F. F. Chehab, P. M. Mannucci, A. B. Federici, and J. E. Sadler. 1987. Gene deletions correlate with the development of alloantibodies in von Willebrand Disease. J. Clin. Invest. 79:1459-1465.

15. Handin, R. I., and D. D. Wagner. 1989. Molecular and cellular biology of von Willebrand factor. Prog. Hematol. 9:233-259.

16. Bonthron, D. T., R. I. Handin, R. J. Kaufman, L. C. Wasley, E. C. Orr, L. M. Mitsock, B. Ewenstein, J. Loscalzo, D. Ginsburg, and S. H. Orkin. 1986. Structure of pre-pro-von Willebrand factor and its expression in heterologous cells. Nature (Lond.). 324:270-273.

17. Shelton-Inloes, B. B., K. Titani, and E. Sadler. 1986. cDNA sequences for human von Willebrand Factor reveal five types of repeated domains and five possible protein sequence polymorphisms. Biochemistry. 25:3164-3171.

18. Fujimura, Y., K. Titani, L. Z. Holland, S. R. Russell, J. R. Roberts, J. H. Elder, Z. M. Ruggeri, and T. S. Zimmerman. 1986. von Willebrand Factor: a reduced and alkylated 52/48-kDa fragment beginning at amino acid residue 449 contains the domain interacting with platelet glycoprotein Ib. J. Biol. Chem. 261:381-385.

19. Fujimura, Y., K. Titani, L. Z. Holland, J. R. Roberts, P. Kostel, Z. M. Ruggeri, and T. S. Zimmerman. 1987. A heparin-binding domain of human von Willebrand factor. J. Biol. Chem. 262:1734-1739.

20. Pareti, F. I., Y. Fujimura, J. A. Dent, L. Z. Holland, T. S. Zimmerman, and Z. M. Ruggeri. 1986. Isolation and characterization of a collegen binding domain in human von Willebrand factor. J. Biol. Chem. 261:15310-15315.

21. Roth, G. J., K. Titani, L. W. Hoyer, and M. J. Hickey. 1986. Localization of binding sites within human von Willebrand factor for monomeric Type III collagen. Biochemistry. 25:8357-8361.

22. De Marco, L., A. Girolami, T. S. Zimmerman, and Z. M. Ruggeri. 1985. Interaction of purified type IIB von Willebrand factor with the platelet membrane glycoprotein Ib induces fibrinogen binding to the glycoprotein IIb/IIIa complex and initiates aggregation. Proc. Natl. Acad. Sci. USA. 82:7424-7428.

23. Saba, H. I., Y. Fujimura, S. R. Saba, A. C. Bruce, G. A. Morelli, Z. M Ruggeri, and T. S. Zimmerman. 1989. Spontaneous platelet aggregation in Type IIB Tampa von Willebrand disease is inhibited by the $52 / 48-\mathrm{kDa}$ fragment of normal von Willebrand factor, which contains the GPIb binding domain. Am. J. Hematol. 30:150-153.

24. Ngo, K. Y., V. T. Glotz, J. A. Koziol, D. C. Lynch, J. Gitschier, P. Ranieri, N. Ciavarella, Z. M. Ruggeri, and T. S. Zimmerman. 1988. Homozygous and heterozygous deletions of the von Willebrand factor gene in patients and carriers of severe von Willebrand Disease. Proc. Natl. Acad. Sci. USA. 85:2753-2757.

25. Peake, I. R., M. B. Liddell, P. Moodie, G. Standen, D. J. Mancuso, E. A. Tuley, L. A. Westfield, J. M. Sorace, J. E. Sadler, C. L. Verweij, and A. L. Bloom. 1990. Severe Type III von Willebrand's disease caused by deletion of Exon 42 of the von Willebrand factor gene: family studies that identify carriers of the condition and a compound heterozygous individual. Blood. 75:654-661.

26. Ginsburg, D., B. A. Konkle, J. C. Gill, R. R. Montgomery, P. L. Bockensted, T. A. Johnson, and A. Y. Yang. 1989. Molecular basis of human von Willebrand disease: analysis of platelet von Willebrand factor mRNA. Proc. Natl. Acad. Sci. USA. 86:3723-3727.

27. Dent, J. A., S. D. Berkowitz, J. Ware, C. K. Kasper, and Z. M. Ruggeri. 1990. Identification of a cleavage site directing the immunochemical detection of molecular abnormalities in type IIA von Willebrand factor. Proc. Natl. Acad. Sci. USA. 87:6306-6310. 
28. Konkle, B. A., and D. Ginsburg. 1988. The addition of endothelial cell growth factor and heparin to human umbilical vein endothelial cell cultures decreases plaminogen activator inhibitor-1 expression. J. Clin. Invest. 82:579585 .

29. Bonthron, D., E. C. Orr, L. M. Mitsock, D. Ginsburg, R. I. Handin, and S. H. Orkin. 1986. Nucleotide sequence of pre-pro-von Willebrand factor cDNA. Nucleic Acids Res. 14:7125-7127.

30. Nichols, W. C., J. J. Liepnieks, V. A. McKusick, and M. D. Benson. 1989 Direct sequencing of the gene for Maryland/German familial amyloidotic polyneuropathy Type II and genotyping by allele-specific enzymatic amplification. Genomics. 5:535-540.

31. Okayama, H., D. T. Curiel, M. L. Brantly, M. D. Holmes, and R. G. Crystal. 1989. Rapid, nonradioactive detection of mutations in the human genome by allele-specific amplification. J. Lab. Clin. Med. 114:105-113.

32. Gyllensten, U. B., and H. A. Erlich. 1988. Generation of single-stranded DNA by the polymerase chain reaction and its application to direct sequencing of the HLA-DQA locus. Proc. Natl. Acad. Sci. USA. 85:7652-7656.

33. Roth, M. S., G. J. Weiner, E. A. Allen, V. H. Terry, C. E. Harnden, M Boehnke, M. S. Kaminski, and Ginsburg D. 1990. Molecular characterization of anti-idiotype antibody-resistant variants of a murine B cell lymphoma. J. Immunol. 145:768-777.

34. Roth, M. S., J. H. Antin, E. L. Bingham, and D. Ginsburg. 1990. Use of polymerase chain reaction-detected sequence polymorphisms to document engraftment following allogeneic bone marrow transplantation. Transplantation. 49:714-720.

35. Mohri, H., Y. Fujimura, M. Shima, A. Yoshioka, R. A. Houghten, Z. M. Ruggeri, and T. S. Zimmerman. 1988. Structure of the von Willebrand Factor domain interacting with glycoprotein Ib. J. Biol. Chem. 263:17901-17094.

36. Barker, D., M. Schafer, and R. White. 1984. Restriction sites containing CpG show a higher frequency of polymorphism in human DNA. Cell. 36:131138.

37. Mohri, H., A. Yoshioka, T. S. Zimmerman, and Z. Ruggeri. 1989. Isolation of the von Willebrand factor domain interacting with platelet glycoprotein Ib, Heparin, and Collagen and characterization of its three distinct functional sites. J. Biol. Chem. 264:17361-17367.

38. Marti, T., S. J. Rosselet, K. Titani, and K. A. Walsh. 1987. Identification of disulfide-bridged substructures within human von Willebrand factor. Biochemistry. 26:8099-8109. 